\title{
A prospective real-world analysis of erenumab in refractory chronic migraine
}

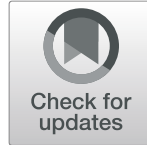

Giorgio Lambru' ${ }^{1}$, Bethany Hill ${ }^{1}$, Madeleine Murphy ${ }^{1}$, Ivona Tylova ${ }^{1}$ and Anna P. Andreou ${ }^{1,2^{*}}$ (D)

\begin{abstract}
Background: Clinical trials have shown the safety and clinical superiority of erenumab compared to placebo in chronic migraine (CM). The aim of this analysis is to evaluate the effectiveness and tolerability of erenumab in a real-world setting in patients with refractory $\mathrm{CM}$.

Methods: This is a prospective single centre real-world audit conducted in patients with CM with and without medication overuse, refractory to established preventive medications, who received monthly erenumab for 6 months.
\end{abstract}

Results: Of 164 patients treated, 162 patients (female $=135$, mean age $46 \pm 14.3$ years old) were included in the audit. Patients had failed a mean of 8.4 preventive treatments at baseline and $91 \%$ of patients failed Botulinum toxin type $\mathrm{A}$ at baseline. The mean reduction in monthly migraine days was 6.0 days at month $3(P=0.002)$ and 7.5 days at month $6(P<0.001)$ compared to baseline. The mean reduction in monthly headache days was 6.3 days $(P<0.001)$ at month 3 and 6.8 days $(P<0.001)$ at month 6 . At month 3,49\%, 35\% and $13 \%$ and at month $6,60 \%$, $38 \%$ and $22 \%$ of patients obtained at least a $30 \%, 50 \%$ and $75 \%$ reduction in migraine days, respectively. The percentage of patients with medication overuse was reduced from $54 \%$ at baseline to $20 \%$ at month 3 and to $25 \%$ at month 6. Compared to baseline, the mean reduction of Headache Impact Test- 6 score was 7.7 points at month 3 (from $67.6 \pm 0.4$ to $59.9 \pm 0.9)(P<0.001)$ and of 7.5 points at month $6(60.1 \pm 1.3)(P=0.01)$. The percentage of patients with severe headache-related disability (HIT-6: 60-78) was reduced from $96 \%$ at baseline to $68 \%$ after three monthly treatments and to $59 \%$ after six treatments. At least one side effect was reported by $48 \%$ of patients at month 1,22\% at month 3 and $15 \%$ at month 6 . Constipation (20\%) and cold/flu-like symptoms (15\%) were the most frequent adverse events reported.

Conclusion: Erenumab may be an effective and well tolerated therapy for medically refractory CM patients with and without medication overuse.

Keywords: Erenumab, Chronic migraine, Refractory migraine, CGRP, Monoclonal antibodies

\section{Introduction}

Chronic migraine affects $1.4-2.2 \%$ of the general population with an annual incidence among episodic migraine people of $2.5 \%[1,2]$. The combination of daily or nearly daily head pain, other comorbidities, namely psychiatric,

\footnotetext{
* Correspondence: anna.andreou@headache-research.com

'The Headache Centre, Pain Management and Neuromodulation Centre, Guy's and St Thomas NHS Foundation Trust, London, UK

${ }^{2}$ Headache Research-Wolfson CARD, Institute of Psychology, Psychiatry and Neuroscience, King's College London, London, UK
}

sleep and pain related, along with the frequent association with medication overuse headache $(\mathrm{MOH})$, contributes to the high degree of socioeconomic burden typical of this condition [3]. The cornerstone strategy to reduce symptoms in $\mathrm{CM}$ includes preventive treatments, however a significant minority of CM patients fails to respond or tolerate numerous preventive treatments [4]. Refractory CM is still a debated definition, but it essentially refers to the group of difficult-to-treat $\mathrm{CM}$ patients who fail to respond/tolerate at least two or three classes/

(c) The Author(s). 2020 Open Access This article is licensed under a Creative Commons Attribution 4.0 International License, which permits use, sharing, adaptation, distribution and reproduction in any medium or format, as long as you give appropriate credit to the original author(s) and the source, provide a link to the Creative Commons licence, and indicate if changes were made. The images or other third party material in this article are included in the article's Creative Commons licence, unless indicated otherwise in a credit line to the material. If material is not included in the article's Creative Commons licence and your intended use is not permitted by statutory regulation or exceeds the permitted use, you will need to obtain permission directly from the copyright holder. To view a copy of this licence, visit http://creativecommons.org/licenses/by/4.0/ The Creative Commons Public Domain Dedication waiver (http://creativecommons.org/publicdomain/zero/1.0/) applies to the data made available in this article, unless otherwise stated in a credit line to the data. 
medications amongst the ones considered effective in migraine prevention [5, 6]. A recent consensus of the European Headache Federation (EHF) has proposed a distinction of the difficult-to-treat migraine patients into resistant, for those who fail to respond or tolerate three drug classes with established evidence in migraine; and refractory, for those patients who fail all drug classes with established evidence in migraine [7]. This group of patients suffers with severe disruption of their quality of life and the refractoriness of their symptoms contributes to high degree of healthcare resources utilization $[8,9]$. A vast unmet need for novel effective and well tolerated preventive treatments remains for these patients.

Monoclonal antibodies (MABs) targeting the calcitonin gene related peptide (CGRP) or its receptor, have been approved by the Food and Drug Administration (FDA) for the prevention of symptoms in episodic and chronic migraine in adults [10-12]. However, to date, there is sparse real-world data on the efficacy of the CGRP MABs, especially in the medically refractory group of chronic migraine patients.

In September 2018 Erenumab (Aimovig ${ }^{\mathrm{Tm}}$ ), a CGRP receptor monoclonal antibody, was made available free-ofcharge in the United Kingdom (UK) for the prevention of $\mathrm{CM}$ in patients who failed at least three preventive treatments, as part of an agreement between Novartis and the National Health System (NHS) Trusts across the UK. The agreement would allow the treatment of CM patients with Erenumab until the National Institute for Health and Care Excellence (NICE) published the outcome of their application appraisal. In September 2019, NICE UK decided not to recommend the use of erenumab in the NHS and since then no more new patients were allowed to be treated according to this scheme. $\mathrm{Pa}-$ tients who had already started the treatment could continue free-of-charge for further 3 years under the agreement. Here we report our experience using erenumab for refractory $\mathrm{CM}$ patients under the above mentioned scheme.

\section{Methods}

This is a registered prospective clinical audit evaluating the effectiveness, safety and tolerability of erenumab in adults with refractory CM. It was part of a service evaluation conducted at the Headache Service at Guy's and St Thomas' NHS Foundation Trust, London, UK. New patients were included in the audit between October 2018 and September 2019.

\section{Participants}

Adult patients meeting the International Headache Society (IHS) criteria for CM who failed at least three preventive treatments were included in the audit [13]. These treatments belonged to the following classes: beta-blockers (propranolol, atenolol), tricyclics (amitriptyline and nortriptyline), anticonvulsants (topiramate, gabapentin, pregabalin and sodium valproate), angiotensin II receptor blocker (candesartan), botulinum toxin type A (BoNT/A), bilateral greater occipital nerve blocks (GONBs) calcium channels blockers (flunarizine), serotonin antagonists (pizotifen), serotonin and norepinephrine reuptake inhibitors (SNRI) namely venlafaxine and duloxetine, other antidepressants (mirtazapine) and noninvasive neuromodulation therapies (single pulse transcranial magnetic stimulation).

Treatment failure was defined as treatment discontinuation due to unacceptable side effects and/or absence of reduction in headache frequency, duration or severity after administration of a preventive medication for at least 12 weeks. Contraindicated treatments were not considered as treatment failures. For patients who underwent a trial with botulinum toxin type A (BoNT/ A), failure to obtain at least $30 \%$ reduction in headache days after two sets of injections was considered treatment failure as per NICE UK guidance [14]. Patients with $\mathrm{MOH}$ were not excluded from the audit, since they constitute a significant proportion of difficult-to-treat $\mathrm{CM}$ patients in real world settings. When medication overuse was present, withdrawal attempts using outpatients pharmacological and non-pharmacological strategies were tried. Patients were allowed to continue oral preventive medications during treatment with Erenumab.

Audit under current national guidelines does not require research ethics committee review (http:// www. hra-decisiontools.org.uk/research/).

\section{Audit design}

Figure 1 outlines the treatment audit profile. Patients were trialled on erenumab for a total of six months before establishing efficacy. All patients received at least three $70 \mathrm{mg}$ Erenumab injections performed one month apart, with the option to increase the dose to $140 \mathrm{mg}$ for further three months if meaningful improvement was not achieved. This treatment paradigm was based upon the lack of definite indications on superiority of $140 \mathrm{mg}$ over $70 \mathrm{mg}$ monthly dose of erenumab, on the most appropriate length of exposure to erenumab in the refractory migraine population and on the high level of refractoriness of our patients. Furthermore, given that our refractory $\mathrm{CM}$ population included predominantly BoNT/A non-responders and that under the NICE guidelines two BoNT/A treatments three months apart are recommended before assessing treatment efficacy, it was decided that a 6-month erenumab trial would have fairly assessed erenumab effectiveness in such a complex population. 


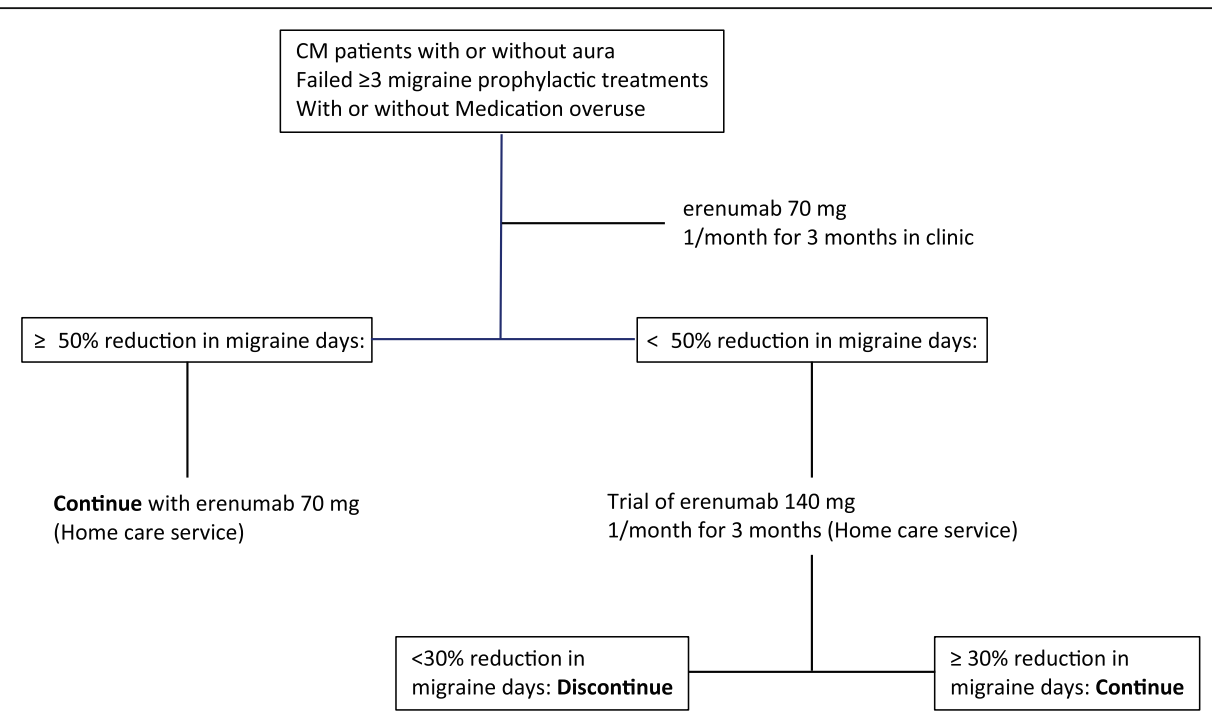

Fig. 1 Audit design: Chronic migraine patients who failed at least 3 preventive treatments, with or without medication overused, were offered monthly subcutaneous injections of erenumab at $70 \mathrm{mg}$ for 3 months. At the three-month time point, patients who achieved at least a $50 \%$ reduction in migraine days, were offered the option to continue their treatment with monthly injections of erenumab at $70 \mathrm{mg}$. Patients who achieved less than $50 \%$ reduction in their migraine days, were offered the option to receive monthly injections of erenumab at 140 mg for the next three consecutive months. Any patient who achieved less than $30 \%$ reduction in their migraine days at the six-month time point discontinued the erenumab treatment, while patients who achieved at least 30\% reduction in their migraine days continue the erenumab treatment at $140 \mathrm{mg}$

Patients were demonstrated how to use the pre-filled autoinjector and the subcutaneous injection was administered or supervised by our headache specialist nurses for the first three visits. Blood pressure (BP) and heart rate were measured in clinic at baseline and at every visit for the first three months. Subsequently, delivery of the medication was arranged via a homecare provider. Patients were asked to check their BP every month for the subsequent treatments. Patients were fully informed about the lack of safety data on erenumab during pregnancy. Female patients were asked to promptly inform the clinic in the event of a pregnancy.

\section{Outcome measures}

A migraine-specific diary and the Headache Impact Test6 (HIT-6) score were used to capture efficacy and disability measures. Patients were required to produce a baseline headache diary and HIT-6 score for at least one month prior to treatment initiation and to continue filling the headache diary on a daily basis along with HIT-6 scores every month for the duration of the trial. Data were entered in an electronic macro database for analysis.

The main efficacy outcomes were changes from baseline in the mean monthly migraine days (MMD) at month 3 and month 6 . Secondary efficacy outcomes included: changes from baseline in mean monthly headache days (MHD), change in mean monthly headachefree days, $30 \%-50 \%-75 \%$ responders (patients who reported respectively a $30 \%-50 \%-75 \%$ reduction in mean
MMD). A "headache day" was defined as a day with headache lasting for $\geq 4 \mathrm{~h}$ and with a severity of $\geq 4 / 10$ on a verbal rating scale $(0$, no head pain, 10 worst pain ever experienced). A "migraine day" was defined according to the IHS classification criteria [13]. A "headache-free day" was defined as a day without any head pain. Changes in abortive treatment intake days and change in the proportion of patients with $\mathrm{MOH}$ were also evaluated. An "abortive treatment intake day" was considered any day where patients consumed abortive treatments for attempted headache relief. An analysis of outcomes in those patients who increased the dose from $70 \mathrm{mg}$ to $140 \mathrm{mg}$ after the first three months was also performed. The cut-off outcome for free-of-charge treatment continuation was reduction in the mean MMD of at least 30\% after six monthly injections. To assess whether any change in efficacy measures was associated with improvement in headache-related disability, change in HIT-6 score were analysed.

Patients were asked about the development of adverse events (AEs) in clinic every month for the first three months, and during telephone follow-ups or clinical appointments for the subsequent months. Adverse events were graded as mild, moderate and severe. The efficacy of erenumab in treated patients was assessed by our specialised headache team every three months for the duration of the trial.

\section{Statistical analysis}

All outcomes pre- and post- erenumab treatment were measured on a continous scale. For all measures 
considered here, data demonstrated a skewed distribution with a significant deviation from normal distribution (Kolmogorov-Smirnov test; $P<0.05$ ). In order to express long term data as a linear graph, skewed data were reflected and transformed to make them more normally distributed $[15,16]$. ANOVA for repeated measures was used to compare the change in values over time. Paired t-test was used to compare any time point against baseline data. Independent t-test was used for independent group comparisons. All data are provided as mean ( \pm standard error), unless stated otherwise.

\section{Results}

\section{Demographic and baseline headache characteristics}

A total of 164 patients received at least one injection of erenumab at $70 \mathrm{mg}$ during the audit period. Completed headache diaries and HIT-6 for all months, were obtained by 162 patients [135 female; mean age 46 Standard Deviation $(\mathrm{SD}) \pm 14$ years] who were included in the analysis. At the time of analysis for this report, 100 patients received three erenumab injections and $73 \mathrm{pa}-$ tients received six injections. Demographic and clinical characteristics of the patients' group at baseline are summarised in Table 1. All patients were medically refractory according to the European headache federation (EHF) consensus [5], with the average number of failed preventive treatments being $8.4 \pm 3$.6. All patients failed to obtain a meaningful response to greater occipital nerve blocks (GONBs). A proportion of $91.4 \%$ of patients had also failed to respond to BoNT/A. Forty-one percent of patients reported a daily headache pattern at baseline with no headache-free days. The vast majority

Table 1 Demographic and clinical characteristics at baseline of refractory chronic migraine patients treated with Erenumab

\begin{tabular}{ll}
\hline & Total number of patients \\
\hline Sex, M/F & $27 / 135$ \\
Age (y), mean \pm SD & $46 \pm 14.3$ \\
CM duration (y), mean \pm SD & $13 \pm 11.9$ \\
Aura, N (\%) & $53(33 \%)$ \\
Medication overuse, N (\%) & $87(54 \%)$ \\
& Mean \pm St. Error \\
Migraine days & $19.7 \pm 0.7$ \\
Headache days & $23.4 \pm 0.6$ \\
Headache free days & $3.7 \pm 0.4$ \\
Abortive treatment intake days & $11.5 \pm 0.7$ \\
HIT-6 score & $67.6 \pm 0.4$ \\
Number of preventive treatments failed & $8.4 \pm 3.6$ \\
BoNT/A non-responders & $148(91.4 \%)$ \\
\hline
\end{tabular}

BoNTA: onabotulinum toxinA, CM, chronic migraine; $F$, female; HIT-6, headache impact test-6; $\mathbf{M}$, male; $\mathbf{N}$, number; $\mathbf{Y}$, years of patients (95.7\%) were classified in the severe impact category at baseline (HIT-6 score: 60-78).

\section{Efficacy outcomes at month 3 and month 6}

Overall, during the entire 6-month observation period post-treatment initiation, MMD days and MHD were significantly reduced compared to baseline (MMDs: $F_{4.8}$, $321.3=3.7, P=0.003$; MHDs: $\left.F_{4.9}, 326.8=3.5, P=0.005\right)$ as well as the number of abortive treatment intake days $\left(F_{3.6,240.1}=4.5, P=0.002\right)$. Additionally, the HIT-6 score was significantly reduced across the entire observational period $\left(F_{3.8,116.6}=3.8, P=0.007\right)$ (Fig. 2$)$.

Compared to baseline, the mean reduction in MMD at month 3 was 6.0 days (from $19.7 \pm 0.7$ to $13.7 \pm 1.0$; $P=$ 0.002 ) and at month 6 was 7.5 days (from $19.7 \pm 0.7$ to $12.2 \pm 1.5, P<0.001)$. The mean reduction in MHD was 6.3 days at month 3 (from $23.4 \pm 0.6$ to $17.1 \pm 1.0 ; P<$ 0.001 ) and 6.8 days at month 6 (from $23.4 \pm 0.6$ to $16.6 \pm 1.6, P<0.001$ ) (Fig. 2). Treatment with erenumab also increased the number of headache-free days by $4.2 \pm 1.0$ days at month $3(P<0.001)$ and by $3.0 \pm 1.4$ days at month $6(P=0.007)$. The proportion of patients with a daily headache pattern was reduced from $41 \%$ at baseline, to $22 \%$ after three and to $21 \%$ after six treatments with erenumab. The mean reduction in abortive treatment days was statistically significant at month $3(3.3 \pm$ $0.7, P=0.01)$ but not at month $6(4.0 \pm 3.1, P=1.1)$.

At month 3, 49\%, 35\% and 13\% out of the 100 patients obtained at least a $30 \%, 50 \%$ and $75 \%$ reduction MMD, respectively. At month $6,60 \%, 38 \%$ and $22 \%$ of the 73 patients obtained at least a $30 \%, 50 \%$ and $75 \%$ reduction in MMD, respectively (Fig. 3). No patient became completely migraine/headache-free during the treatment.

A single dose of erenumab $70 \mathrm{mg}$ led to a conversion from a CM pattern to an episodic pattern in $27 \%$ of our refractory $\mathrm{CM}$ patients. At month 3, the percentage of patients displaying an episodic migraine pattern was 39\% and at month 6, it was $40 \%$ (Fig. 4).

At month $6,60 \%$ of patients $(N=44 / 73)$ achieved at least 30\% reduction in mean MMD and therefore continued the treatment. Conversely, 40\% ( $N=29$ patients) did not reach the continuation threshold, hence the treatment was discontinued.

\section{Dose comparison}

In 53 patients (53\%) who did not achieve a clinically significant improvement with three monthly erenumab $70 \mathrm{mg}$ injections, the dose was increased to $140 \mathrm{mg}$ for the subsequent three months. Of these, 43/53 completed the 6 months treatment at the time of the analysis. Compared to baseline, $16 / 43$ patients obtained $>30 \%$ but $<50 \%$ reduction in mean MMD and 27/43 patients obtained $<30 \%$ reduction in mean MMD at month 3. At month 6, erenumab at $140 \mathrm{mg}$ significantly decreased mean MMD 


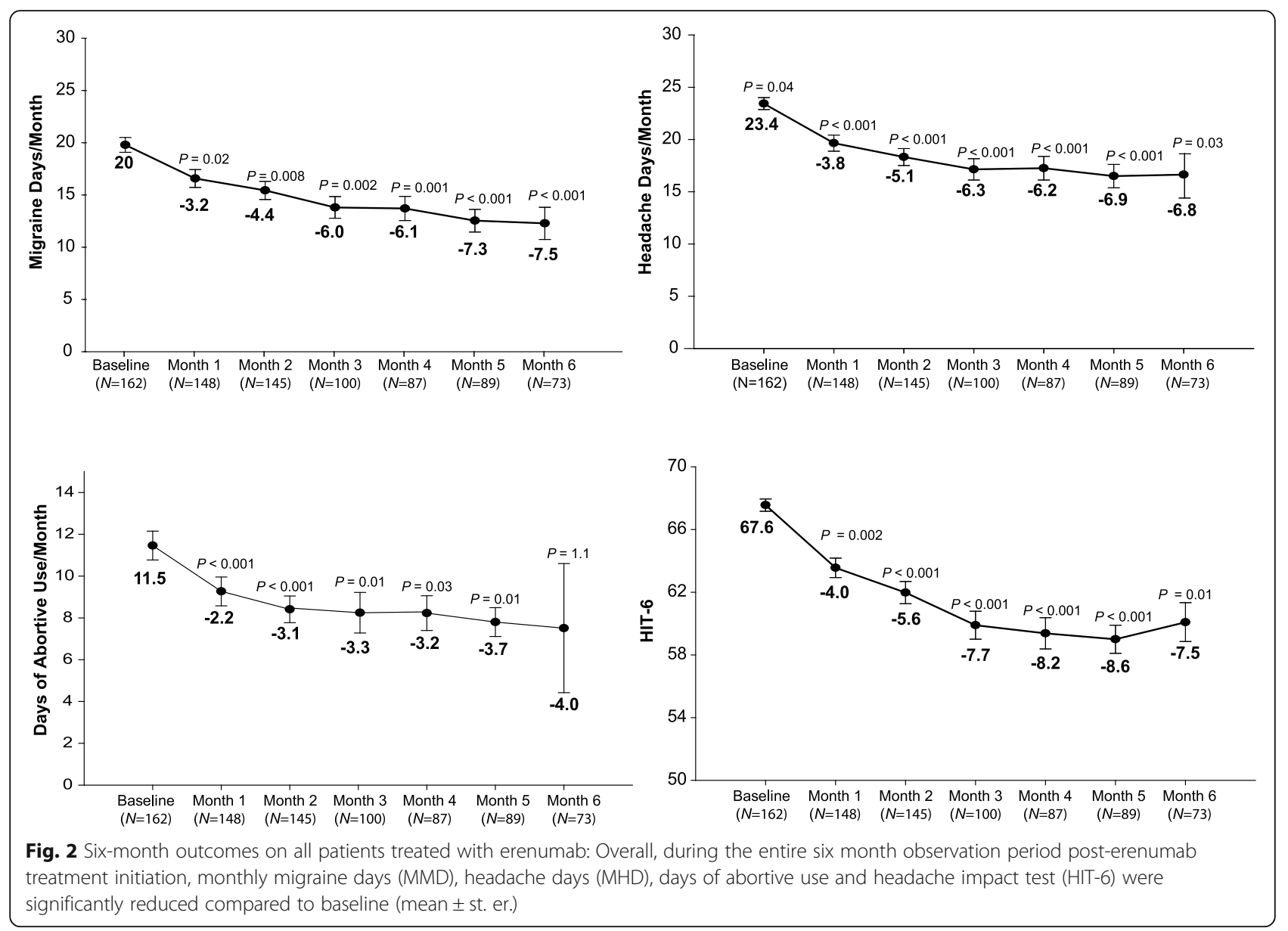

$(-3.6$ vs $-9.8 ; P<0.001)$ and mean MHD $(-4.1$ vs 9.2; $P=0.003)$, compared with data collected at month 3 after 3-monthly injections of Erenumab at $70 \mathrm{mg}$. Switching to $140 \mathrm{mg}$ had no additional effect on headache free days $(+2.2 \mathrm{vs}+5.5 ; P=0.095)$, on number of abortive treatment intake days $(-3.8$ vs -4.9 ;
$P=0.25)$ or on HIT-6 scores $(-2.8$ vs $-3.9 ; P=0.47)$. However, of the 27 patients who did not achieve a $30 \%$ reduction in mean MMD after three monthly injections with erenumab $70 \mathrm{mg}$, five patients (19\%) achieved at least a $30 \%$ reduction in mean MMD at month 6, after increasing the dose to $140 \mathrm{mg}$ for

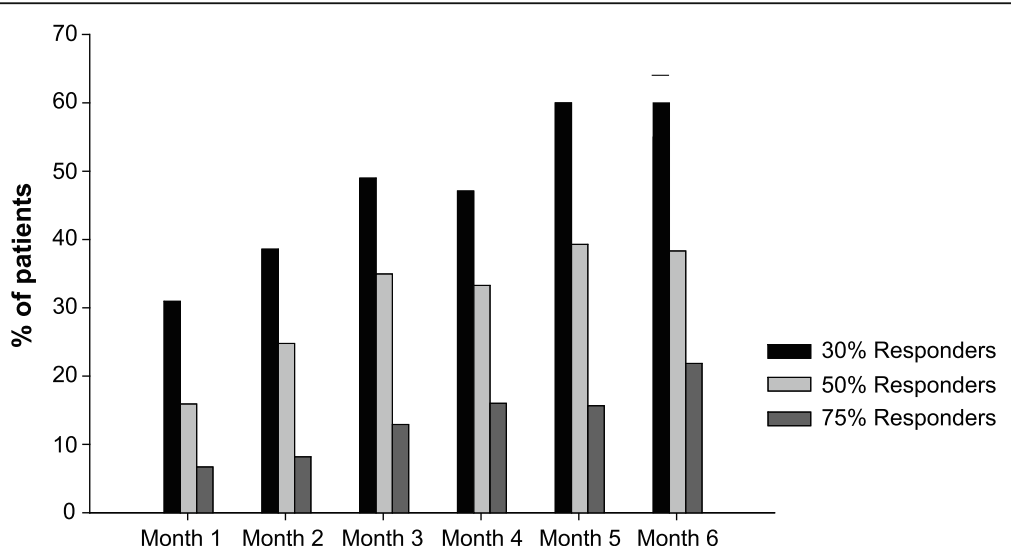

Fig. 3 Monthly responders rates: Percentage of patients who achieved at least a $30 \%, 50 \%$ or $75 \%$ reduction in monthly migraine days per month, post-erenumab treatment initiation 


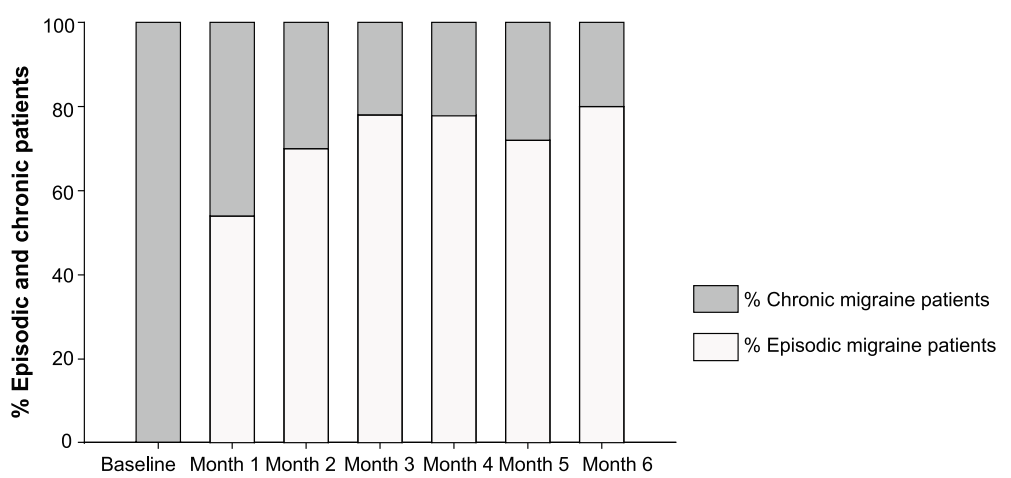

Fig. 4 Percentage of chronic and episodic migraine patients: Percentage of patients who converted into an episodic migraine pattern $(<15$ headache days/month) at each month, post-erenumab treatment initiation

three consecutive months and hence continued the treatment further.

\section{Comparison of $\mathrm{MOH}$ and non-MOH patients}

No significant differences in number of MMD, MHD and headache-free days emerged between the group of patients with $\mathrm{MOH}$ and that without $\mathrm{MOH}$ at baseline (Table 2). The percentage of patients with $\mathrm{MOH}$ was reduced from $54 \%$ at baseline to $20 \%$ after three treatment and to $25 \%$ after 6 treatments. Patients characterised as $\mathrm{MOH}$ at baseline had no significant differences in the reduction of migraine and headache days or HIT-6 score compared to non-medication overuse patients $(\mathrm{nMOH})$ following three and six monthly injections of erenumab (Table 2).

\section{Headache-related disability}

Compared to baseline, the reduction of mean HIT-6 score was 7.7 points at month 3 (from $67.6 \pm 0.4$ to $59.9 \pm 0.9)(P<0.001)$ and of 7.5 points at month 6 $(60.1 \pm 1.3)(P=0.01)$. The percentage of patients with severe headache-related disability was reduced from $96 \%$ at baseline to $68 \%$ after three monthly treatments and to $59 \%$ after six treatments. A percentage of $29 \%$ after three monthly treatments and $22 \%$ after six treatments reported some or little/ headache-related impact (Table 3).

\section{Safety and tolerability}

After one injection $48 \%(N=77 / 162)$ of patients reported at least one side effect. Of them, the most frequent adverse events were constipation in 32 patients (42\%) and flu/coldlike symptoms in 25 patients (32\%). Twenty-two percent of patients $(N=22 / 100)$ at month 3 and $15 \%(N=11)$ at month 6 reported at least one adverse. Table 4 outlines the changes of incidence of adverse events overtime in the whole population. Adverse events were transient, lasting up to two weeks post-injection and described as mild or moderate in the great majority of patients. However, $12 \%$ of patients $(N=19)$ discontinued erenumab due to severe adverse events, eight during months $1-3$ and nine during months 4-6. The reasons for discontinuation were: severe

Table 2 Clinical characteristics at baseline and after three and six treatments with erenumab for the patients who presented with medication overuse $(\mathrm{MOH})$ and non-medication overuse $(\mathrm{nMOH})$ at baseline, before treatment initiation

\begin{tabular}{|c|c|c|c|c|c|c|}
\hline & \multicolumn{2}{|l|}{ Baseline } & \multicolumn{2}{|l|}{ Month 3} & \multicolumn{2}{|l|}{ Month 6} \\
\hline & $\overline{\mathrm{MOH}}$ & $\mathrm{nMOH}$ & $\overline{\mathrm{MOH}}$ & $\mathrm{nMOH}$ & $\overline{\mathrm{MOH}}$ & $\mathrm{nMOH}$ \\
\hline \multirow[t]{2}{*}{ Migraine days } & $19.0 \pm 0.9$ & $19.9 \pm 1.2$ & $12.4 \pm 1.2^{*}$ & $14.7 \pm 1.8$ & $11.4 \pm 1.3^{*}$ & $13.3 \pm 2.2 \#$ \\
\hline & $P=0.2$ & & $P=0.1$ & & $P=0.1$ & \\
\hline \multirow[t]{2}{*}{ Headache Days } & $23.2 \pm 0.7$ & $23.8 \pm 0.8$ & $15.3 \pm 1.3^{*}$ & $19.5 \pm 1.6 \#$ & $15.7 \pm 1.4^{*}$ & $18.2 \pm 2.0$ \\
\hline & $P=0.3$ & & $P=0.1$ & & $P=0.1$ & \\
\hline \multirow[t]{2}{*}{ Crystal clear headache days } & $4.6 \pm 0.6$ & $2.7 \pm 0.6$ & $9.4 \pm 1.2^{*}$ & $5.7 \pm 1.4 \#$ & $8.2 \pm 1.4^{*}$ & $4.5 \pm 1.5$ \\
\hline & $P=0.1$ & & $P=0.3$ & & $P=0.1$ & \\
\hline \multirow[t]{2}{*}{ Days consumed abortives } & $17.9 \pm 0.7$ & $4.0 \pm 0.4$ & $10.3 \pm 1.0^{*}$ & $4.4 \pm 0.6$ & $9.7 \pm 1.1^{*}$ & $3.8 \pm 0.7$ \\
\hline & $P<0.001$ & & $P=0.1$ & & $P=0.001$ & \\
\hline \multirow[t]{2}{*}{ HIT-6 } & $66.7 \pm 1.0$ & $66.7 \pm 1.0$ & $58.3 \pm 1.3^{*}$ & $60.2 \pm 2.2 \#$ & $53.4 \pm 3.1^{*}$ & $62.1 \pm 4.3$ \\
\hline & $P=0.8$ & & $P=0.2$ & & $P=0.4$ & \\
\hline
\end{tabular}

*P $<0.05$ compared to baseline values in $\mathrm{MOH}$ patients 
Table 3 Changes in HIT-6 headache disability categories after three and six erenumab treatments

\begin{tabular}{llll}
\hline & Baseline $(\boldsymbol{N}=162) \boldsymbol{N}(\%)$ & Month 3 $(\boldsymbol{N}=100) \boldsymbol{N}(\%)$ & Month 6 (N=73) $\boldsymbol{N}(\%)$ \\
\hline Severe impact (60-78) & $156(96 \%)$ & $68(68 \%)$ & $43(59 \%)$ \\
Substantial impact (56-59) & $5(3 \%)$ & $3(3 \%)$ & $14(19 \%)$ \\
Some impact (50-55) & $1(1 \%)$ & $15(15 \%)$ & $11(16 \%)$ \\
Little or no impact (<48) & $0(0 \%)$ & $14(14 \%)$ & $5(6 \%)$ \\
\hline
\end{tabular}

HIT-6, headache impact test-6; N, number

constipation in nine patients, severe and consistent headache worsening after each injection in five patients, severe flu-like symptoms in two patients, whole body itchiness in one patient, severe mood deterioration in one patient and new onset hypertension in one patient. This patient developed raised BP while on monthly erenumab $70 \mathrm{mg}$ and then $140 \mathrm{mg}$, confirmed with a 24-h BP monitoring and a cardiology assessment. A thorough cardiac work-up did not show any other underlying causes. Although a $75 \%$ migraine improvement was reported, the treatment was discontinued and the BP normalised within six weeks. No other patients demonstrated pathological changes to $\mathrm{BP}$ or heart rate during the first six months of treatment. One patient became pregnant during the first four months of treatment, hence the treatment was discontinued. Pregnancy was reported without complications.

\section{Discussion}

This is the first large, independent, prospective analysis evaluating the effectiveness and tolerability of erenumab in real-world $\mathrm{CM}$ patients with and without $\mathrm{MOH}$, refractory to medical treatments. Refractory $\mathrm{CM}$ is a very disabling migraine variant; it often represents a medical challenge for headache specialists and poses substantial burden on healthcare service utilisation [8]. The vast majority of patients treated in this audit would largely meet the recently EHF updated criteria for refractory CM since they failed all the drug classes with evidence in migraine prevention including injectable treatments and often non-invasive neuromodulation approaches, had severe migraine symptoms and reported high levels of headache-related disability [7]. Furthermore, a significant proportion of patients displayed a chronic daily headache pattern at baseline.

The results of this report suggested that over a period of six months, erenumab was well tolerated and effective in preventing migraine symptoms. Compared to baseline, erenumab led to a significant improvement across all the efficacy outcomes, which was sustained throughout the six months and led to a relevant reduction in headache-related disability. Our efficacy outcomes were less impressive than the ones of a recent real-life open-label study conducted predominantly CM patients [17]. Indeed, at month 6, 69\% and $62 \%$ of patients obtained respectively at least $30 \%$ and $50 \%$ reduction in MMD. Similar outcomes were observed in the BoNT/A non-responder subgroup analysis. Possible explanation for the outcome differences between studies may include patients selection. In the Italian study, patients failed 2-4 treatments, hence were considered difficult-totreat, whereas in our study most patients failed all established treatments, hence were more refractory to medical treatments. Furthermore, the increased proportion of responders at month 6 in the Italian study may have been influenced by the fact that non-responders could have discontinued the treatment earlier, whereas in our analysis, all patients, apart from those who discontinued because of adverse events, continued for the trial for six month, even if they did not respond at month 3 .

The month-3 reduction in MMD with erenumab 70 mg reported in our analysis was similar to the main endpoint of the pivotal phase $2 \mathrm{CM}$ clinical trial both when the whole study population was considered but also

Table 4 Percentage of incidences of adverse events at months 1, 3 and 6 following treatment with erenumab in the overall population of patients treated with erenumab

\begin{tabular}{llll}
\hline & Month 1 $(\boldsymbol{N}=162) \boldsymbol{N}(\%)$ & Month 3 $(\boldsymbol{N}=100) \boldsymbol{N}(\%)$ & $\begin{array}{c}\text { Month 6 } \\
(\boldsymbol{N}=73) \boldsymbol{N}(\%)\end{array}$ \\
\hline Constipation & & & $4(5 \%)$ \\
Cold-flu/like & $32(20 \%)$ & $11(11 \%)$ & $2(3 \%)$ \\
Generalised aches/pain & $25(15 \%)$ & $8(8 \%)$ & $1(1 \%)$ \\
Itchiness & $10(6 \%)$ & $1(1 \%)$ & $1(1 \%)$ \\
Injection site reaction (pain/skin redness) & $8(5 \%)$ & $0(0 \%)$ & $1(1 \%)$ \\
Muscle spasms & $5(3 \%)$ & $0(0 \%)$ & $0(0 \%)$ \\
Others & $3(2 \%)$ & $4(4 \%)$ & $3(4 \%)$ \\
\hline
\end{tabular}

$\mathbf{N}$, number 
when the subgroup of patients who failed at least two preventive treatments was analysed [18, 19]. Furthermore, the $50 \%$ response rate with erenumab $70 \mathrm{mg}$ in the overall Phase 2 trial population was $40 \%$ and in the subgroup analysis of patients with at least two prior treatment failures was $35.6 \%$, very similar to the $35 \%$ response rate found in our patients. At month 6, a progressive improvement in most of the efficacy measures was observed in our patients, possibly due to the longer exposure to erenumab, but perhaps also due to the increased dose which may have enhanced the clinical improvement in some of our patients. A similar effect was reported in the 1-year open-label extension of the pivotal phase 2 clinical trial [20]. However, in that study, the withdrawal of treatment non-responders may have biased the results by impacting positively on the outcomes, whereas in our audit all patients were treated for at least six months unless they decided to discontinue it due to side effects.

Reduction of at least $30 \%$ in monthly migraine frequency is considered a clinically meaningful change especially in the refractory migraine population [21, 22]. If this cut-off was applied after three months treatment in our refractory patients, almost half of the patients (49\%) would qualify for treatment continuation with erenumab. However, a small proportion of patients who did not obtain a $30 \%$ reduction in MMD at month 3 , met the $30 \%$ threshold for treatment continuation at month 6 , suggesting that highly refractory CM may benefit from a six month treatment, similarly to BoNT/A recommended regimen, to include those with a delayed response.

Along with the uncertainty about the optimal trial duration in refractory $\mathrm{CM}$, it is also unclear whether the $140 \mathrm{mg}$ erenumab dose is clinically superior to the 70 $\mathrm{mg}$ dose. In patients who switched from $70 \mathrm{mg}$ to 140 mg, we observed a greater improvement in MMD and MHD. Furthermore a significant minority of nonresponders after three monthly $70 \mathrm{mg}$ erenumab injections, became responders once they were switched to the $140 \mathrm{mg}$ dose, indicating a degree of superiority of the dose of $140 \mathrm{mg}$ compared to the $70 \mathrm{mg}$. Similar findings emerged from the post hoc analysis of the pivotal erenumab CM study, which pointed towards a slight superiority of $140 \mathrm{mg}$ dose in patients who failed two or more preventive treatments compared to those who were naïve or failed one treatment only [19], even when they had $\mathrm{MOH}$ [23]. Moreover, during the 1-year open-label treatment extension of the parent study, erenumab 140 mg showed greater clinical benefit compared to the 70 $\mathrm{mg}$ dose in a number of outcomes including reduction in MMD, $50 \%-75 \%-100 \%$ responder rates and reduction in days of use of abortive migraine medications [20]. Our data along with the post-hoc analysis of the pivotal CM trial, suggest that erenumab $140 \mathrm{mg}$ dose may provide greater and more sustained efficacy compared to the $70 \mathrm{mg}$ dose in the difficult-to-treat $\mathrm{CM}$ population.

Erenumab has led to a sustained resolution of $\mathrm{MOH}$ in a meaningful proportion of patients. Our data were similar to the outcome of the subgroup analysis of 274 $\mathrm{CM}$ patients with $\mathrm{MOH}$ treated with erenumab or placebo, showing no significant difference in treatment effect between the group with $\mathrm{MOH}$ and non-MOH [21]. Given that $\mathrm{MOH}$ is frequently diagnosed in tertiary referral clinics, the efficacy of erenumab in this even more complex group of patients, makes it a valuable option also in those patients in whom abortive treatment withdrawal is proven to be difficult to achieve or ineffective.

Erenumab has consistently shown a very favourable safety and tolerability profile across the CM and episodic migraine trials with low discontinuation rates [18, 24-26]. In the subgroup analysis of the pivotal phase 2 clinical trial, patients with prior preventive treatment failure treated with erenumab reported a higher proportion of AEs compared to placebo (42-58\% depending upon the different doses) [19]. Our refractory group of patients displayed a similar proportion of AEs at month 1 (48\%). However, with subsequent treatments, the proportion of patients complaining of AEs diminished substantially, suggesting that longer exposure to erenumab may lead to improved tolerability. Constipation and cold-like symptoms were confirmed to be the most frequent AEs, similarly to the pivotal clinical trials in migraine [18, 24-26]. However, the percentage of patients with constipation observed in our study was higher, especially during the first three months of treatment, compared to the one in the clinical trials. A likely explanation may be that we systematically asked about this adverse event every month for the first three months. However, a higher percentage of constipation (13.5\%) was also observed in a recent real-life study [17], suggesting that the real-world population may be more susceptible to medications' adverse events perhaps in view of their frequent co-morbidities. The discontinuation rate displayed by our patients was greater than the ones reported in previous studies, even when the subgroup analysis of the patients with prior preventive treatments failure was considered [18, 19]. Patients referred to our tertiary Centre have often been previously seen by multiple specialists across the country and tried many pharmacological options. High treatments discontinuation rates in the refractory $\mathrm{CM}$ patients may constitute one of the biological aspects of this complex migraine variant, especially in the context of multiple comorbidities, such as irritable bowel syndrome which was reported by a high proportion of our patients and may at least explain discontinuation due to severe constipation. 
Hypertension has sparsely been associated to erenumab exposure [27]. In our patient no other causes of hypertension were identified. It is likely that their newonset hypertension was erenumab-related given that no other causes were identified, the patient was otherwise healthy at baseline and the BP normalised with discontinuation of erenumab. In light of this case, it may be advisable to counsel patients about this possible and likely very rare $\mathrm{AE}$.

The main limitation of this audit is the open label design. However, it is unlikely that the symptoms improvement could be explained by placebo alone. The strengths of this report are the large number of patients with a refractory form of migraine, which reflects the type of complex and difficult-to-treat patients seen in tertiary headache clinics and the prospective and the real-world nature of the analysis, which includes patients not subject to strict inclusion and exclusion criteria.

Recently published position statements and guidelines have debated patients selection and positioning of antiCGRP MABs within the arsenal of migraine preventive treatments in clinical practice. Despite their meaningful efficacy and good tolerability demonstrated in clinical trials across the spectrum of episodic and chronic migraine, this new class of drugs is costly, hence in clinical practice it may be reserved for the difficult-to-treat/refractory subgroup of $\mathrm{CM}$ patients only, which were largely excluded for the clinical trials $[28,29]$. Our analysis along with another recent study [17], provided the first real-world evidence of efficacy of erenumab in such population, supporting its meaningful role even in the complex difficult-to-treat CM population. In conclusion, erenumab was effective in the prevention of migraine symptoms in our refractory CM patients with and without $\mathrm{MOH}$.

\section{Conclusion}

Erenumab was effective in the prevention of migraine symptoms in our highly refractory CM patients with and without $\mathrm{MOH}$. Erenumab's beneficial effect seems to be sustained, progressive overtime and not influenced by the level of patients' refractoriness. The improvement in migraine symptoms led to a vast reduction in headacherelated disability in our very complex group of patients. Treatment-related AEs reduced overtime, confirming its favourable tolerability profile. Hypertension emerged as a rare $\mathrm{AE}$ that needs to be taken into account when considering this treatment.

\section{Abbreviations}

AEs: Adverse events; BoNT/A: Botulinum toxin type A; BP: Blood pressure; CGRP: Calcitonin gene-related peptide; CM: Chronic migraine; EHF: European Headache Federation; EM: Episodic migraine; FDA: Food and Drug Administration; GONBs: Greater occipital nerve blocks; HIT-6: Headache Impact Test, 6th Edition; ICHD: International Classification of Headache Disorders; IHS: International Headache Society; MABs: Monoclonal antibodies;
MHD: Monthly headache days; MMD: Monthly migraine days; $\mathrm{MOH}$ : Medication overuse headache; NHS: National Health System; NICE: National Institute for Health and Care Excellence; SD: Standard Deviation; SE: Standard error; U.K.: United Kingdom

\section{Acknowledgements}

The authors thank Prof Lawrence Moon, King's College London, for his assistance in the statistical analysis of the data.

\section{Authors' contributions}

$G L$ and $A A$ conceived the audit and its design; $G L, A A, B H, M M$, IT performed the data acquisition; GL and AA performed analysis; All authors performed data interpretation. GL and AA drafted the manuscript; all authors revised and approved the final manuscript.

\section{Funding}

No funding was available for this paper.

\section{Availability of data and materials}

Anonymized data are available from the Authors upon reasonable request.

Ethics approval and consent to participate

Not applicable.

\section{Consent for publication}

Not applicable.

\section{Competing interests}

G.L. has received speaker honoraria, funding for travel and has received honoraria for participation in advisory boards sponsored by Allergan, Novartis, Eli Lilly and TEVA. He has received speaker honoraria, funding for travel from electroCore, Nevro Corp. and Autonomic Technologies. B.H. reports no disclosure. M.M. reports no disclosure. I.T. reports no disclosure. A.P.A received speaker honoraria and funding for travel from Allergan, Eli Lilly and eNeura, honoraria for participation in advisory boards sponsored by Allergan and Eli Lilly, sponsorship for educational purposes from eNeura, Allergan, Autonomic Technologies and Novartis, and an equipment grant from eNeura.

Received: 23 April 2020 Accepted: 19 May 2020

Published online: 01 June 2020

\section{References}

1. Natoli JL, Manack A, Dean B et al (2010) Global prevalence of chronic migraine: a systematic review. Cephalalgia 30:599-609. https://doi.org/10. 1111/j.1468-2982.2009.01941.x

2. Manack AN, Buse DC, Lipton RB (2011) Chronic migraine: epidemiology and disease burden. Curr pain headache rep 15:70- 78. https://doi.org/10.1007/ s11916-010-0157-z

3. Lipton RB, Bigal ME, Diamond $M$ et al (2007) Migraine prevalence, disease burden, and the need for preventive therapy. Neurology 68(5):343-349. https://doi.org/10.1212/01.wnl.0000252808.97649.21

4. Hepp Z, Dodick DW, Varon SF, Gillard P, Hansen RN, Devine EB (2015) Adherence to oral migraine-preventive medications among patients with chronic migraine. Cephalalgia 35:478-488. https://doi.org/10.1177/ 0333102414547138

5. Martelletti $P$, Katsarava Z, Lampl C et al. (2014) Refractory chronic migraine: a consensus statement on clinical definition from the European Headache Federation. J Headache Pain ;28;15:47. doi: https://doi.org/10.1186/11292377-15-47

6. Schulman EA, Lake AE 3rd, Goadsby PJ et al (2008) Defining refractory migraine and refractory chronic migraine: proposed criteria from the refractory headache special interest section of the American headache society. Headache 48:778-782. https://doi.org/10.1111/j.1526-4610.2008. 01132.x

7. Simona Sacco, Mark Braschinsky, Anne Ducros, Christian Lampl, Patrick Little, Antoinette Maassen van den Brink et al (2020) European headache federation consensus on the definition of resistant and refractory migraine. $\checkmark$ Headache Pain

8. Bloudek LM, Stokes M, Buse DC et al (2012) Cost of healthcare for patients with migraine in five European countries: results from the international 
burden of migraine study (IBMS). J headache pain; 13: 361-378. 14. https:// doi.org/10.1007/s10194-012-0460-7

9. Irimia P, Palma JA, Fernandez-Torron R et al (2011) Refractory migraine in a headache clinic population. BMC Neurol 11:94. https://doi.org/10.1186/1471-2377-11-94

10. Markham A (2018) Erenumab: First Global Approval. Drugs 78:1157-1161. https://doi.org/10.1007/s40265-018-0944-0

11. Hoy SM (2018) Fremanezumab: First Global Approval. Drugs 78:1829-1834 https://doi.org/10.1007/s40265-018-1004-5

12. Lamb YN (2018) Galcanezumab: First Global Approval. Drugs. 78:1769-1775. https://doi.org/10.1007/s40265-018-1002-7

13. Headache Classification Committee of the International Headache Society (IHS) (2018) The international classification of headache disorders, 3rd edition. Cephalalgia 38(1):1-211. https://doi.org/10.1177/0333102417738202

14. NICE Technology appraisal guidance. Botulinum toxin type A for the prevention of headaches in adults with chronic migraine https://www.nice. org.uk/guidance/TA260

15. Howell D (2007) Statistical methods for psychology ( $6^{\text {th }}$ ed). Belmont, CA. Thomson Wadsworth

16. Tabachnick BG, Fidel LS (2007) Using multivariant statistics, 5th edn. Allyn and Bacon, Boston

17. Ornello R, Casalena A, Frattale I, Gabriele A, Affaitati G, Giamberardino MA et al (2020) Real-life data on the efficacy and safety of erenumab in the Abruzzo region, Central Italy. J Headache Pain 21(1):32. https://doi.org/10. 1186/s10194-020-01102-9

18. Tepper S, Ashina M, Reuter U, Brandes JL, Doležil D, Silberstein S et al (2017) Safety and efficacy of erenumab for preventive treatment of chronic migraine: a randomised, double-blind, placebo-controlled phase 2 trial. Lancet Neurol 16(6):425-434. https://doi.org/10.1016/S1474-4422(17)30083-2

19. Ashina M, Tepper S, Brandes JL et al (2018) Efficacy and safety of erenumab (AMG334) in chronic migraine patients with prior preventive treatment failure: a subgroup analysis of a randomized, double-blind, placebo-controlled study. Cephalalgia 38:1611-1621. https://doi.org/10.1177/0333102418788347

20. Tepper SJ, Ashina M, Reuter U et al (2020) Long-term safety and efficacy of erenumab in patients with chronic migraine: results from a 52-week, openlabel extension study. Cephalalgia 26. https://doi.org/10.1177/ 0333102420912726

21. Dworkin RH, Turk DC, McDermott MP et al (2009) Interpreting the clinical importance of group differences in chronic pain clinical trials: IMMPACT recommendations. Pain 2009.146(3):238-244. https://doi.org/10.1016/j.pain.08.019

22. Silberstein S, Tfelt-Hansen P, Dodick DW et al (2008) Guidelines for controlled trials of prophylactic treatment of chronic migraine in adults. Cephalalgia 28(5):484-495. https:/doi.org/10.1111/j.1468-2982.2008.01555.X

23. Tepper SJ, Diener HC, Ashina M, Brandes JL, Friedman DI, Reuter U et al (2019) Erenumab in chronic migraine with medication overuse: subgroup analysis of a randomized trial. Neurology. 92(20):e2309-e2e20. https://doi. Org/10.1212/WNL.0000000000007497

24. Goadsby PJ, Reuter U, Hallström Y, Broessner G, Bonner JH, Zhang F et al (2017) A controlled trial of Erenumab for episodic migraine. N Engl J Med 377(22):2123-2132. https://doi.org/10.1056/NEJMoa1705848

25. Dodick DW, Ashina M, Brandes JL, Kudrow D, Lanteri-Minet M, Osipova V et a (2018) ARISE: a phase 3 randomized trial of erenumab for episodic migraine. Cephalalgia. 38(6):1026-1037. https://doi.org/10.1177/ 0333102418759786

26. Reuter U, Goadsby PJ, Lanteri-Minet M, Wen S, Hours-Zesiger P, Ferrari MD et al (2018) Efficacy and tolerability of erenumab in patients with episodic migraine in whom two-to-four previous preventive treatments were unsuccessful: a randomised, double-blind, placebo-controlled, phase 3b study. Lancet. 392(10161): 2280-2287. https//doi.org/10.1016/s0140-6736(18)32534-0

27. Kudrow D, Pascual J, Winner PK et al (2020) Vascular safety of erenumab for migraine prevention. Neurology;4;94(5):e497-e510. https://doi.org/10.1212/ WNL.0000000000008743

28. Sacco S, Bendtsen L, Ashina M, Reuter U, Terwindt G, Mitsikostas DD et al (2019) European headache federation guideline on the use of monoclonal antibodies acting on the calcitonin gene related peptide or its receptor for migraine prevention. J headache pain;20(1):6. https:/doi.org/10.1186/s10194-018-0955-y

29. Tiseo C, Ornello R, Pistoia F, Sacco S (2019) How to integrate monoclonal antibodies targeting the calcitonin gene-related peptide or its receptor in daily clinical practice. J Headache Pain 20(1):49. https://doi.org/10.1186/s10194-019-1000-5

\section{Publisher's Note}

Springer Nature remains neutral with regard to jurisdictional claims in published maps and institutional affiliations.

Ready to submit your research? Choose BMC and benefit from:

- fast, convenient online submission

- thorough peer review by experienced researchers in your field

- rapid publication on acceptance

- support for research data, including large and complex data types

- gold Open Access which fosters wider collaboration and increased citations

- maximum visibility for your research: over $100 \mathrm{M}$ website views per year

At BMC, research is always in progress.

Learn more biomedcentral.com/submissions 